\title{
Computer Supported Test Generation from SDL Specifications
}

\author{
Anders Ek ${ }^{a}$, Jan Ellsberger ${ }^{a}$ and Anthony Wiles ${ }^{b}$ \\ aTeleLogic, P.O. Box 4128, S-203 12 Malmö, Sweden, \\ E:-mail:Anders.Ek@telelogic.se, Jan.Ellsberger@telelogic.se \\ 'Telia Research AB, Ovandal Lagga, S-755 98 Uppsala, Sweden, \\ E-mail:Anthony.Wiles@farsta.trab.se
}

\begin{abstract}
As part of a project at Telia (Ek, Ellsberger and Wiles 1993), a method for test generation has been developed and implemented in a tool acting as a link between the SDT and ITEX (TeleLogic 1993) toolsets. The purpose of the method is to provide tool supported generation of test cases for behaviour tests from SDL specifications (CCITT 1988). The method prevents a test suite designer from creating test cases that are not consistent with the corresponding SDL specification. This paper gives a description of the method together with a description of the tool (STED). Also some experiences from the application of the method on the GSM Data Link protocol LAPDm are reported.
\end{abstract}

\section{INTRODUCTION}

A common approach to automatic test case generation from formal specifications is to first generate the complete set of test cases for a given implementation relation. Test selection is then performed on this set of test cases given certain test selection criterias, e.g. cost and value of the final test suite (Brinksma, Tretmans and Verhaard 1991). The problem with this approach is that the behaviour defined by the specification is typically very large, often infinite, which causes problems during the calculation of the behaviour and the subsequent test selection. Examples of this approach can be found in (Wezeman 1989; Cavalli, Kim and Hiong 1992). ${ }^{1}$

A different approach, more aligned with (ISO-9646 1991), is to perform the test selection prior the generation of test cases. A representation of the selected test purposes are then used as input to the method in order to generate only the test cases that will be part of the final test suite. Examples of this approach can be found in (van de Burgt, Kroon and Peeters 1991; Grabowski, Hogrefe and Nahm 1993).

In this paper we present the latter approach applied to SDL specifications. The test purposes are expresses as sequences of events and the tests are expressed in TTCN.(ISO-9646 1991). How to perform test selection, i.e. identification of the test purposes, is out of scope of the method. Within a project at Telia (Ek, Ellsberger

1. A comprehensive overview and comparison of different test generation techniques can be found in (FMCT 1994). 
and Wiles 1993), the method has been implemented in a tool (STED), integrated as a link between the SDT and ITEX tool sets (TeleLogic 1993). The STED tool was used in a case study to derive tests for the GSM Data Link protocol. Compared to other existing methods, the method presented in this paper imposes very small restrictions on the specifications from which tests are generated.

The rest of this paper is organised as follows: Section 2 gives a description of the method and section 3 gives an overview of the STED tool. In section 4 we report on the experiences from using the tool on the GSM Data Link protocol specification. Finally in section 5 we draw some conclusions.

\section{DESCRIPTION OF THE METHOD}

The method supports the test designer by preventing him from creating test cases that are not consistent with the behaviour of the corresponding requirements specification. Input to the method is an SDL specification of the IUT together with the selected test purposes, and the output is TTCN declarations (PCO, ASP and type declarations) and a set of TTCN test cases. The different steps of the method are illustrated in fig. 1.

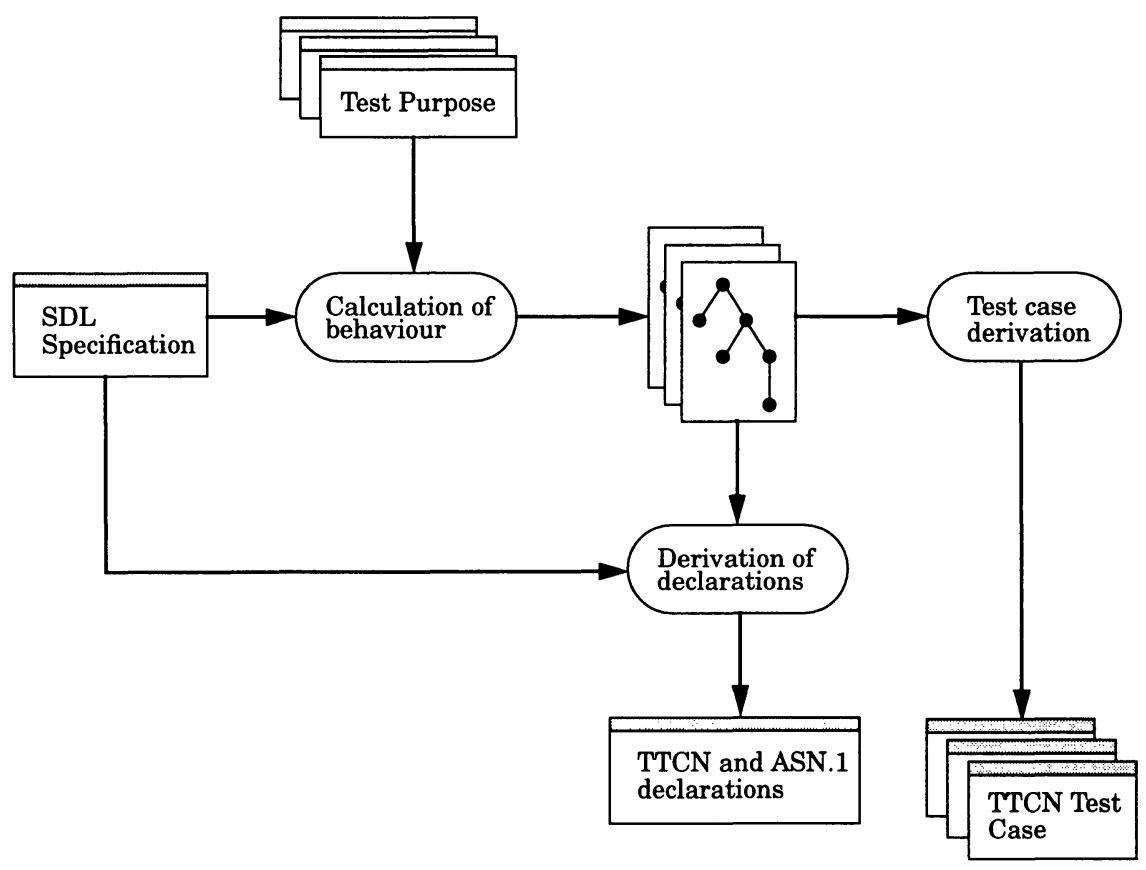

Figure 1. An overview of the method. 


\subsection{SDL Specifications}

The SDL specifications used as input to the method must reflect the test method for which tests will be generated, i.e. the PCOs must correspond to channels connected to the environment in the SDL specification, and the signals conveyed by such channels must correspond to ASPs (or PDUs). For example, an SDL specification of the Inres protocol (FMCT 1994) is depicted in fig. 2.

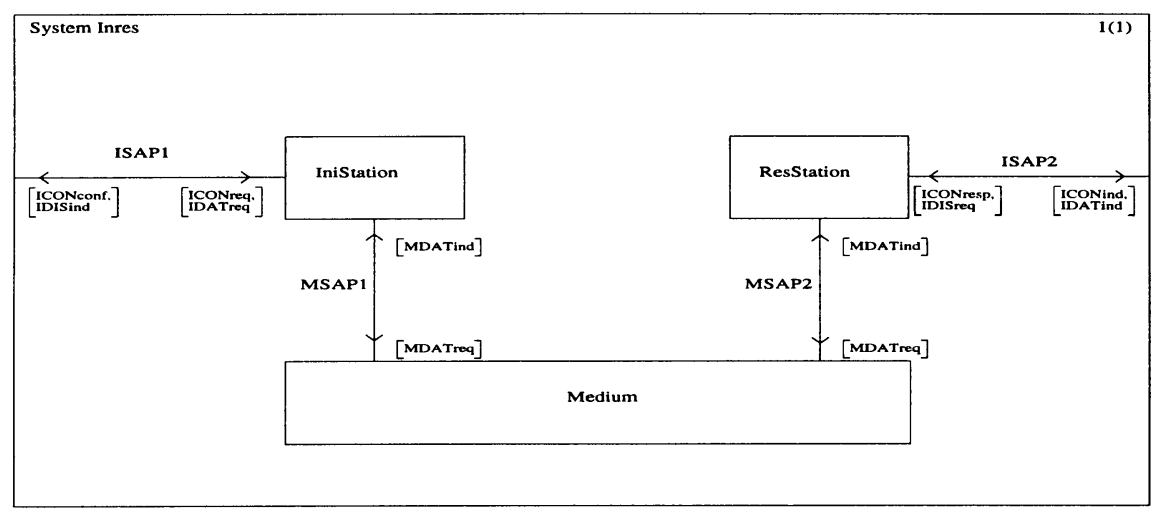

Figure 2. SDL specification of the Inres protocol.

If this specification is to be used for the generation of tests for the local test method where the IUT is the Initiator side, then the SDL specification has to be changed such that the channels ISAP1 and MSAP2 corresponds to PCOs and the block IniStation and the block Medium corresponds to the SUT. This is accomplished by simply removing the block ResStation and connecting the channel MSAP2 to the environment, as depicted in fig. 3.

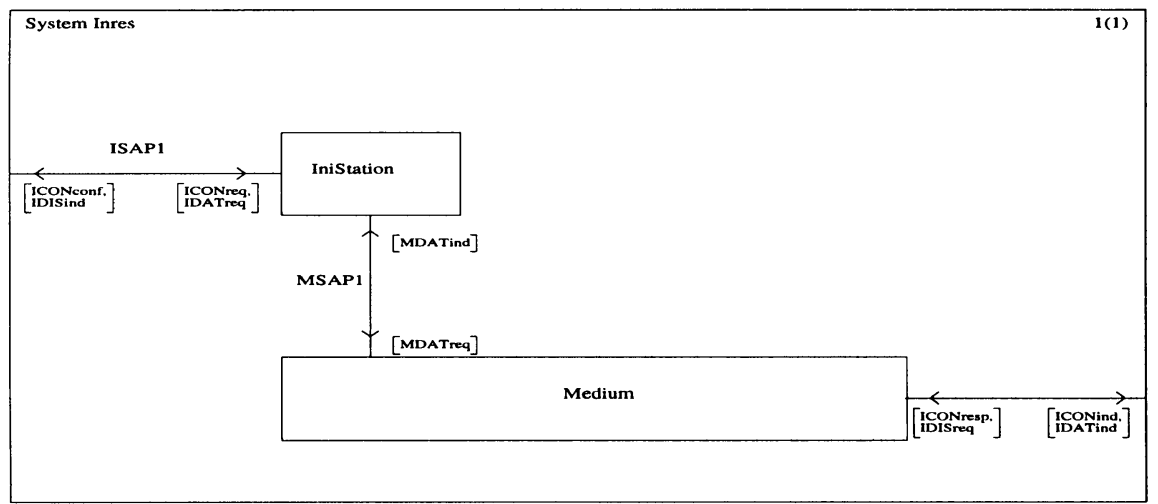

Figure 3. SDL specification of the Inres protocol used for the generation of tests for the Initiator side. 
The data types of the parameters of signals conveyed by channels connected to the environment, i.e. the ASPs or PDUs, is mapped to ASN.1 type declarations in the test suite. Therefore, only the following SDL data types are allowed in signal declarations of signals corresponding to ASPs or PDUs:

- Boolean

- Integer

- Charstring

- Enumerated types

- $\quad$ syntypes of Boolean, Integer and Charstring

- $\quad$ structured type declarations using Struct

- Arrays, where the elements are of any of the types above

For all other data type declarations in the SDL specification, no restrictions apply.

\subsection{Test Purposes}

According to (ISO-9646 1991) a test purpose is the starting point for the creation of test cases, and a test purpose is given as a short informal description of the intention of a test case. In general, test purposes are given in terms of some functionality of the related functionality, for example "Test connection establishment" etc.

In our approach, a test purpose is given as the sequence of events ("Test Purpose (TP) event sequence" (van de Burgt, Kroon and Peeters, 1991)) that constitutes some functionality of the related specification. For example, a TP event sequence for the connection establishment of the Inres protocol in fig. 3 can be given as:

Send ICONreq from UT

Receive MDATind(CR, 0,0$)$ at $\mathrm{LT}$

Send MDATreq $(\mathrm{CC}, 0,0)$ from LT

Receive ICONconf at UT

This TP event sequence can then be formalised using an MSC, as depicted in fig. 4.

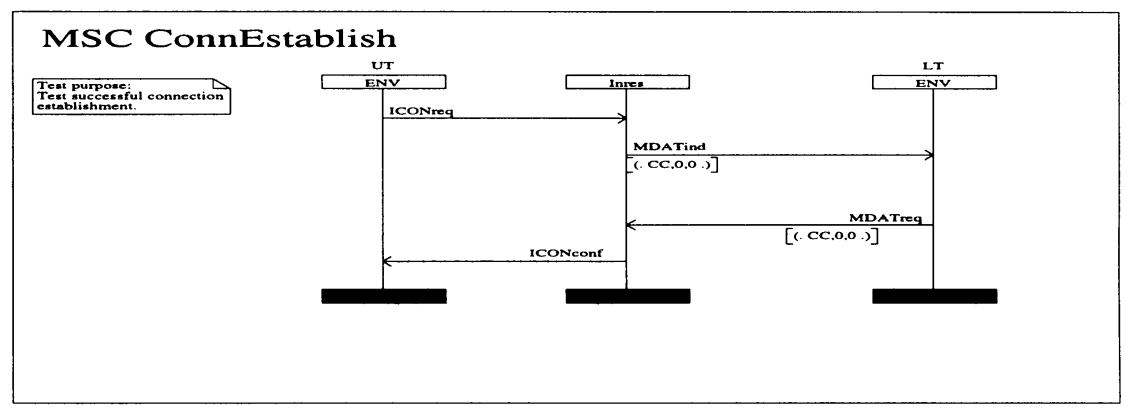

Figure 4. A TP event sequence formalised using an MSC. 
Since the SDL specification contains the SUT, the TP event sequence is the sequence of events that take place in the environment (ENV) of the SDL specification. Using the tool SDT, MSCs can be automatically generated during a simulation of the SDL specification.

\subsection{Derivation of TTCN Test Cases}

A TP event sequence describes the sequence of events (trace) that should lead to a PASS verdict in the corresponding test case. What has to be added to the TP event sequence in order to be a complete test case is the additional traces that should lead to INCONCLUSIVE verdicts. These traces are calculated given the TP event sequence as input. For example, the traces of the Inres protocol (fig. 3) given the TP event sequence of fig. 4 as input is as depicted in fig. 5 .

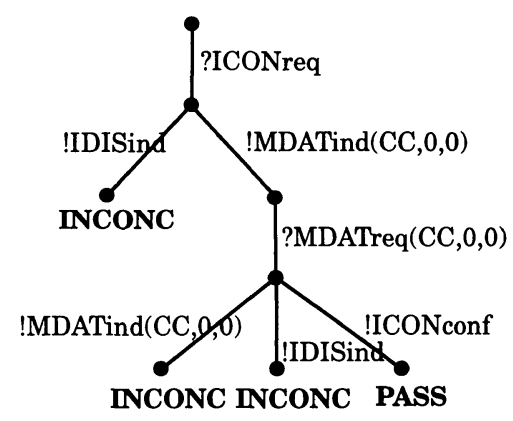

Figure 5. The behaviour of the Inres protocol related to the test purpose of fig. 4. Only the externally observable events are shown.

TTCN test cases are then derived from the calculated behaviour. This translation is straightforward, input events are mapped to TTCN output events and output events are mapped to TTCN input events. PCO references are derived from the channel specifications in the SDL specification. Constraint references are derived from the values of the SDL signal parameters. The TTCN input constraints are given in the TP event sequence, and the TTCN output constraints are calculated when calculating the traces.

Traces in the test case that should lead to a FAIL verdict are handled by default test steps $\mathrm{PCO}_{\mathrm{n}}$ ?OTHERWISE FAIL introduced in the final TTCN test suite.In this way the semantics of the verdicts in the derived TTCN test cases is:

PASS The behaviour of the implementation matches the TP event sequence.

INCONC The behaviour of the implementation does not match the TP event sequence, but is part of the set of traces of the SDL specification. 
FAIL The behaviour of the implementation is not part of the set of traces of the SDL specification.

Hence, when a FAIL verdict is given for an implementation, the implementation is not trace equivalent with the specification.

\subsection{Derivation of TTCN Declarations}

The derivation of TTCN declarations is based on the declarations in the SDL specification. The following table shows how the derivation is performed:

\begin{tabular}{|l|l|l|}
\hline SDL & TTCN & Note \\
\hline $\begin{array}{l}\text { Channel connected to the } \\
\text { environment }\end{array}$ & PCO declaration & The PCO role is not derived \\
\hline $\begin{array}{l}\text { Signal type declaration of } \\
\text { signals conveyed by chan- } \\
\text { nels connected to the envi- } \\
\text { ronment }\end{array}$ & ASP declaration & $\begin{array}{l}\text { If a signal type should be } \\
\text { mapped to an ASP or PDU } \\
\text { declaration cannot be deter- } \\
\text { mined based only on the } \\
\text { information in the SDL } \\
\text { specification }\end{array}$ \\
\hline $\begin{array}{l}\text { Type declarations of param- } \\
\text { eters of signals correspond- } \\
\text { ing to ASPs: }\end{array}$ & ASN.1 type declarations & \\
\hline Boolean & Boolean & \\
\hline Integer & Integer & \\
\hline Character & IA5String & \\
\hline Charstring & IA5String & \\
\hline Enumerated types & Enumeration & \\
\hline $\begin{array}{l}\text { structured type declara- } \\
\text { tions using Struct }\end{array}$ & Sequence & \\
\hline Arrays & Sequence Of & \\
\hline $\begin{array}{l}\text { Values of parameters of sig- } \\
\text { nals conveyed by channels } \\
\text { connected to the environ- } \\
\text { ment }\end{array}$ & Constraint declarations & \\
\hline
\end{tabular}

\section{TOOL SUPPORT}

The method has been implemented in a tool STED (SDL-based TTCN Editor) which establishes a link between the SDT (SDL) and ITEX (TTCN) tools, offering an integrated SDL-TTCN environment.

The ITEX tool contains a tabular test case editor that supports the graphical TTCN notation (fig. 6). When linked to the STED tool, the editing can be performed in two modes: Unsynchronised or Synchronised. In the unsynchronised mode the editor works as a conventional TTCN editor. When in synchronised mode the editor provides the user with knowledge about the behaviour of the corresponding SDL specification.This is accomplished in the following way: 
The user can add a TTCN Send statement to the test case. This is a manual step where the user defines the constraint to be associated with the send statement. Then, by issuing a "Receive" command, the editor automatically generates all valid responses from the SUT and adds them as TTCN Receive statements to the test case. Also the constraint references and the verdicts (PASS or INCONC) to the receive statements are automatically generated. Also constraint declarations are automatically generated each time a new constraint is referenced.

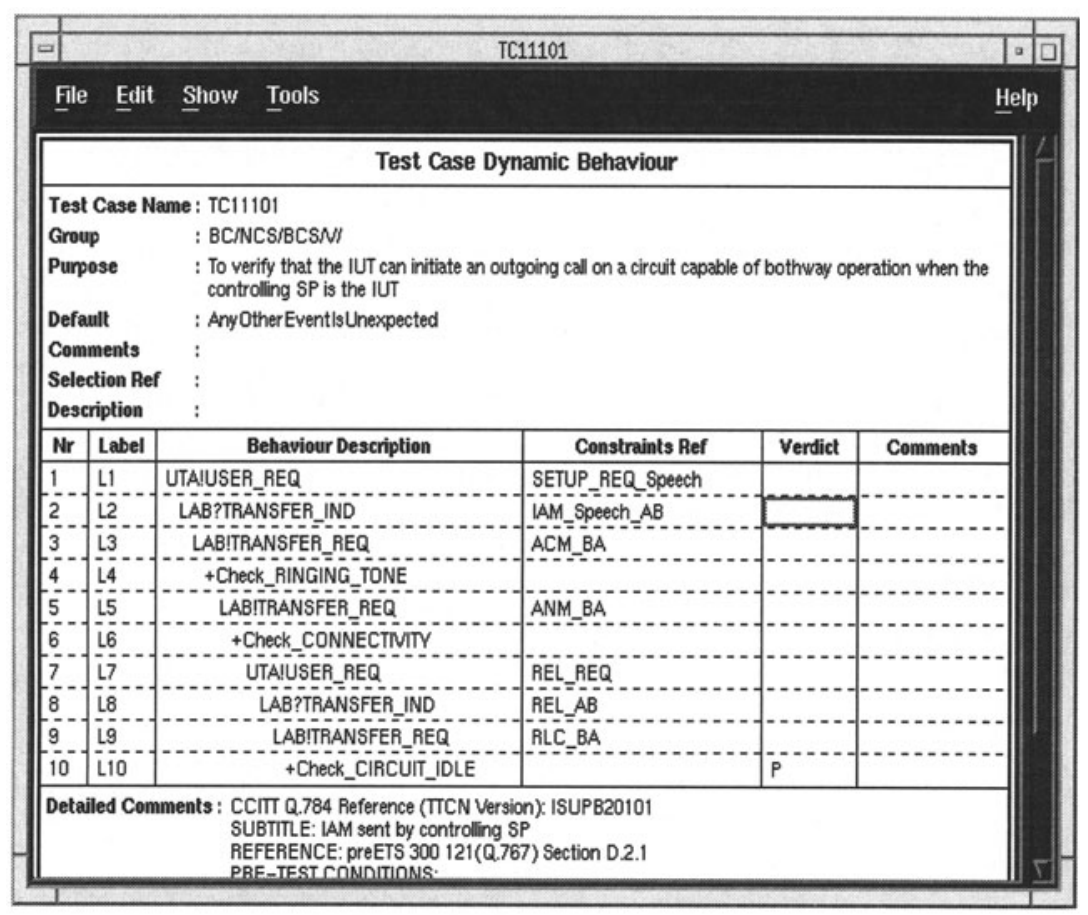

Figure 6. The user interface to the ITEX test case editor.

Finally, by issuing a "Generate declarations" command, the PCO, ASP and type declarations are generated and included in the test suite.

The STED tool also offers the user access to the SDL specification via the SDT tool. For example, by selecting a line in the test case it is possible to "pop up" an SDL editor with the SDL symbols corresponding to the selected test case line highlighted. It is also possible to access e.g. variable values, signal queues etc. of the corresponding SDL system. 


\section{CONCLUSIONS}

The STED tool was used to produce a TTCN test suite for the GSM Data Link protocol LAPDm. The SDL specification of LAPDm consists of approximately 450 pages of SDL diagrams. The English language test purposes for the test cases was taken from an existing test suite for the same protocol and expressed as MSCs. The test cases produced using STED was compared with the corresponding manually produced test cases. In a few cases errors were found in the manually produced test cases.

The estimated time savings (in percent) when producing a test suite using STED compared to manual production, was found to be:

$\begin{array}{ll}\text { Static declarations } & 80 \% \\ \text { Constraints } & 50 \% \\ \text { Behaviour } & 50 \%\end{array}$

In general, the cost of abstract test suite production should be reduced by at least $40 \%$ when using STED. Note however that this estimation does not take the cost of producing an SDL specification into account. An SDL specification will probably not be developed only to be used as a basis for test generation. An SDL specification is also used for automatic validation, as a basis for implementation etc.

Since the user of the STED tool is working in a TTCN environment, no expert knowledge of SDL is required. This, together with that the tool includes protocol knowledge directly available to the user, means that the education time can be kept to a minimum.

\section{REFERENCES}

Brinksma, E., J. Tretmans and L. Verhaard. (1991). “A Framework for Test Selection”, in Proceedings of 11th International Symposium on Protocol Specification, Testing and Verification (PSTV), Stockholm, Sweden.

Cavalli, A., S. U. Kim and P. K. Hiong (1992). "Generation of a Finite State Machine from a LOTOS Specification of INRES Protocol using CAESAR”, Institut National des Télécommunications (INT), Evry, France.

CCITT. (1988). "Specification and Description Language (SDL), Recommendation Z.100", Blue book.

Ek, A., J. Ellsberger and A. Wiles. (1993). "Experiences with Protocol Specification using SDL”, in Proceedings of the 6th IWPTS, Pau, France.

FMCT. (1994). "Formal Methods in Conformance Testing", ISO SC.21 P.54/ITU-T SG.10 Q.8, Working draft, March 1994, Madrid.

Grabowski, J., D. Hogrefe and R. Nahm. (1993). "Test Case Generation with Test Purpose Specification by MSCs", in Proceedings of 6th SDL Forum, Darmstadt, Germany.

ISO-9646. (1991). "Information Technology - Open Systems Interconnection - Conformance Testing Methodology and Framework", International Standard, International Organisation for Standardisation (ISO).

van de Burgt, S. P., J. Kroon and A. M. Peeters. (1991). "Interactive test generation from LOTOS specifications", Partner position, P.RNL.RNLAP042, RACE Project SPECS (1046).

Wezeman, C. (1989). “The CO-OP Method for Compositional Derivation of Canonical Testers", in Proceedings of 9th International Symposium on Protocol Specification, Testing and Verification (PSTV). 